\title{
Reducing the risk of infection in a 74-year-old man who is to receive prednisone
}

\author{
Daniel M. Shafran MD, Paul E. Bunce MD, Wayne L. Gold MD
}

A 74-year-old man presents with a one-month history of cutaneous bullae. Skin biopsy confirms a diagnosis of bullous pemphigoid. His dermatologist plans to start treatment with prednisone $(40 \mathrm{mg} / \mathrm{d}$ for four weeks followed by a tapering regimen) and considers potential infectious complications of the therapy and ways to screen for and prevent them.

\section{Should this patient be screened for latent tuberculosis?}

A retrospective case-control study in the United Kingdom involving 497 patients with tuberculosis (TB) who had no history of HIV infection or cancer reported an odds ratio of 7.7 (95\% confidence interval [CI] 2.8-21.4) for TB developing in patients taking $15 \mathrm{mg}$ or more of prednisone per day (or equivalent). ${ }^{1}$ Citing this study, the Canadian Thoracic Society advises that patients receiving glucocorticoid therapy at a dose equivalent to $15 \mathrm{mg}$ or more of prednisone per day for one month or more are at moderate risk of reactivation of TB and recommends screening with a tuberculin skin test (sensitivity $90 \%$, specificity $>95 \%$, false-positive rate $\leq 5 \%$ ) or an interferon-gamma release assay (sensitivity $80 \%-90 \%$, specificity $>95 \%$, false-positive rate $\leq 5 \%)^{2}{ }^{2}$ However, a costeffectiveness analysis found that screening for latent TB was cost-effective only if the risk of disease was high. ${ }^{3}$ Therefore, it is reasonable to screen patients prescribed $15 \mathrm{mg}$ or more of prednisone per day for one month or more who are at high risk of latent TB (Appendix 1, available at www.cmaj .ca/lookup/suppl/doi:10.1503/cmaj.131430/-/DC1). Patients with a positive test result should receive treatment. $^{2}$

\section{Does this patient require screening} for chronic hepatitis B virus infection?

Patients receiving $7.5 \mathrm{mg}$ or more of prednisone per day should be screened for hepatitis B virus (HBV) infection. Manifestations of HBV reactivation range from asymptomatic elevations in HBV DNA viral load and alanine aminotrans- ferase level to acute hepatic failure. The risk of $\mathrm{HBV}$ reactivation appears to be minimal in patients receiving less than $7.5 \mathrm{mg}$ of prednisone per day, because there are no published reports of HBV reactivation at this dosage. ${ }^{4}$ Reactivation has occurred in patients with rheumatic diseases who received lower doses of steroids in combination with other immunosuppressive medications. ${ }^{4}$

Most of the literature regarding HBV reactivation in patients receiving immunosuppressive therapies is based on studies involving oncology patients; less is known about patients being treated for other conditions. In a retrospective cohort study that included more than 10000 patients undergoing chemotherapy, HBV reactivation occurred in $23 \%$ of patients with a positive result for hepatitis B surface antigen (HBsAg) or hepatitis B core antibody (anti-HBc). ${ }^{5}$ All-cause mortality was significantly lower among patients who received HBV prophylaxis $(22 \%)$ than among those who did not $(71 \%)$.

Canadian guidelines recommend using the HBsAg test to screen for chronic HBV infection in all patients receiving immunosuppressive therapies. ${ }^{6}$ American guidelines recommend using both the HBsAg and anti-HBc tests. ${ }^{7} \mathrm{How}-$ ever, a cost-effectiveness analysis found that universal screening for HBV using both tests in patients with nonhematopoietic solid tumours was not cost-effective. ${ }^{8}$ It is therefore reasonable to screen all patients receiving $7.5 \mathrm{mg}$ or more of prednisone per day with the $\mathrm{HBsAg}$ test alone and to add screening for anti-HBc in patients who will receive multiple immunosuppressants.

Patients with positive test results for $\mathrm{HBsAg}$ should be referred to a specialist for measurement of their HBV DNA viral load and antiviral prophylaxis, ideally before the start of immunosuppressive therapy (Appendix 1). ${ }^{8}$

\section{Should this patient be screened for infection with Strongyloides stercoralis?} Strongyloides stercoralis is a nematode endemic to many tropical and subtropical regions. Screening for $S$. stercoralis infection is recommended for all
Competing interests: None declared.

This article has been peer reviewed.

Correspondence to: Wayne Gold, wayne.gold@uhn.ca CMAJ 2014. DOI:10.1503 /cmaj.131430 
patients prescribed immunosuppressive therapies who have lived in or travelled to an endemic area, regardless of the time lapsed or the anticipated duration of glucocorticoid therapy (Appendix 1). Strongyloides hyperinfection syndrome has a mortality approaching $100 \%$ and has been associated with short courses of glucocorticoid therapy in patients who are infected. ${ }^{9}$ In a retrospective casecontrol study, glucocorticoid use was associated with a relative risk of 3.28 (95\% CI 1.24-10.43) for disseminated strongyloidiasis. ${ }^{10}$ Stool microscopy is the most specific test, but it has poor sensitivity. Serologic testing has a high negative predictive value, making it useful for ruling out infection (Appendix 1). ${ }^{9}$ Patients with positive test results should be treated with ivermectin in consultation with an infectious disease specialist.

\section{Should this patient receive prophylaxis against Pneumocystis jirovecii pneumonia?}

Prophylaxis with trimethoprim-sulfamethoxazole should be considered for patients receiving the equivalent of $20 \mathrm{mg}$ or more of prednisone per day for four to eight weeks or more, based on expert opinion. ${ }^{11}$ In a retrospective analysis of 116 HIV-negative patients with a first episode of Pneumocystis jirovecii pneumonia, $90.5 \%$ of the patients had received glucocorticoid therapy during the month before onset. One-quarter received as little as $16 \mathrm{mg}$ of prednisone per day, and onequarter received less than eight weeks of therapy. ${ }^{11}$ There are no guidelines for prophylaxis against $P$. jirovecii pneumonia in patients with dermatologic or rheumatologic conditions who are receiving glucocorticoid therapy. However, a systematic review and meta-analysis of 12 randomized controlled trials involving immunocompromised patients who were HIV-negative reported a $91 \%$ reduction in the occurrence of $P$. jirovecii pneumonia when trimethoprimsulfamethoxazole was administered (relative risk $0.09,95 \%$ CI $0.02-0.32) .{ }^{12}$ Adverse events that necessitated discontinuation of the trimethoprimsulfamethoxazole occurred in $3.1 \%$ of adults and resolved after the treatment was stopped. Atovaquone or dapsone may be used in patients who cannot tolerate trimethoprim-sulfamethoxazole. ${ }^{12}$

\section{Is this patient at risk of other infections related to glucocorticoid therapy?}

Patients receiving glucocorticoid therapy are at increased risk of infection with viral, bacterial and fungal pathogens. The Canadian National Advisory Committee on Immunization recommends administration of all age-appropriate vaccines and boosters before initiation of immunosuppressive therapy. ${ }^{13}$ Although recommendations for reduc- ing the risk of infection rely on the best available evidence, there still is a need for high-quality data showing the efficacy and safety of these recommendations in these populations. If febrile illness develops during treatment, an infectious cause should be strongly suspected.

\section{Case revisited}

Further inquiry showed that the patient had lived in China for two years 20 years ago. Before the prednisone treatment is started, a tuberculin skin test is performed. Testing for HBsAg is also requested, as well as serology and stool microscopy for S. stercoralis infection.

The results of the tuberculin skin test and the tests for S. stercoralis infection are negative. The HBsAg test result is positive. The patient is referred to a specialist in liver diseases, who prescribes lamivudine for prophylaxis against HBV reactivation. Because the patient will receive more than $20 \mathrm{mg}$ of prednisone per day for at least one month, prophylaxis for $P$. jirovecii pneumonia will be considered at the one-month follow-up visit.

\section{References}

1. Jick SS, Lieberman ES, Rahman MU, et al. Glucocorticoid use, other associated factors, and the risk of tuberculosis. Arthritis Rheum 2006;55:19-26.

2. Canadian Thoracic Society. Canadian tuberculosis standards, 7th edition. Can Resp J 2013;20(Suppl A):1-174.

3. Oxlade O, Schwartzmann K, Menzies D. Interferon-gamma release assays and TB screening in high-income countries: cost effectiveness analysis. Int J Tuberc Lung Dis 2007;11:16-26.

4. Calabrese LH, Zein NN, Vassilopoulos D. Hepatitis B virus reactivation with immunosuppressive therapy in rheumatic diseases: assessment and prevention strategies. Ann Rheum Dis 2006; 65:983-9.

5. Hwang J, Fisch M, Zhang H, et al. Reactivation of hepatitis B infection among patients with cancer. Hepatology 2011;54 (Suppl 4):445A

6. Coffin CS, Fung SK, Ma MM. Management of chronic hepatitis B: Canadian association for the study of the liver consensus guidelines. Can J Gastroenterol 2012;26:917-38.

7. Lok ASF, McMahon BJ. AASLD Practice Guideline update Chronic hepatitis B: update 2009. Hepatology 2009;50:661-2.

8. Day FL, Karnon J, Rischin D. Cost-effectiveness of universal hepatitis B virus screening in patients beginning chemotherapy for solid tumors. J Clin Oncol 2011;29:3270-7.

9. Ramanathan R, Nutman T. Strongyloides stercoralis infection in the immunocompromised host. Curr Infect Dis Rep 2008;10:105-10.

10. Davidson RA, Fletcher RH, Chapman LE. Risk factors for strongyloidiasis: a case-control study. Arch Intern Med 1984;144:321-4.

11. Yale SH, Limper AH. Pneumocystis carinii pneumonia in patients without acquired immunodeficiency syndrome: associated illness and prior corticosteroid therapy. Mayo Clin Proc 1996;71:5-13.

12. Green H, Paul M, Vidal L, et al. Prophylaxis of pneumocystis pneumonia in immunocompromised non-HIV-infected patients: systematic review and meta-analysis of randomized controlled trials. Mayo Clin Proc 2007;82:1052-9.

13. Canadian immunization guide. Ottawa: Public Health Agency of Canada; 2013. Available: www.phac-aspc.gc.ca/publicat/cig-gci /index-eng.php (accessed 2013 Dec. 11).

Affiliations: Department of Medicine (Shafran, Bunce, Gold), University of Toronto; Division of Infectious Diseases (Bunce, Gold), University Health Network, Toronto, Ont.

Contributors: All of the authors contributed to the preparation of this manuscript. All approved the final version submitted for publication and have agreed to act as guarantors of the work. 